\title{
Brief Report: The Dopamine-3-Receptor Gene (DRD3) is Associated with Specific Repetitive Behavior in Autism Spectrum Disorder (ASD)
}

\author{
Wouter G. Staal • Mariken de Krom • \\ Maretha V. de Jonge
}

Published online: 21 June 2011

(c) The Author(s) 2011. This article is published with open access at Springerlink.com

\begin{abstract}
Recently the DRD3 gene has been associated with ASD in two independent samples. Follow up analysis of the risk allele of the SNP rs167771 in 91 subjects revealed a significant association with a specific type of repetitive behavior: the factor "insistence on sameness" (IS) derived from the Autism Diagnostic Interview. This risk allele was associated with a decreased risk for IS, but not with any other symptomatology. Further study and replication of this finding is necessary, bearing in mind that these results would not be statistically significant if corrected for multiple testing.
\end{abstract}

Keywords DRD3 $\cdot$ rs167771 $\cdot$ Autism $\cdot$ Stereotyped behavior

\section{Introduction}

Stereotyped and repetitive behaviors are core symptoms of autism spectrum disorders (ASD) and form an essential part of the classification criteria for ASD as described in the

\section{W. G. Staal (ه)}

Karakter, Radboud University Nijmegen Medical Centre

(Cognitive Neuroscience), Reinierpostlaan 12,

6525 CG Nijmegen, The Netherlands

e-mail:w.staal@karakter.com

\section{M. de Krom}

Rudolf Magnus Institute of Neuroscience, Department of Neuroscience and Pharmacology, UMC Utrecht,

Utrecht, The Netherlands

\section{V. de Jonge}

Rudolf Magnus Institute of Neuroscience, Department of Child and Adolescent Psychiatry, University Medical Center Utrecht, Utrecht, The Netherlands preliminary draft revisions to the current DSM-IV(TR) for DSM-V (see www.dsm5.org). These behaviors cause severe impairment in functioning and stereotyped and repetitive behaviors are one of the main reasons for pharmacological interventions in children with ASD (Parikh et al. 2008).

The neurobiology of repetitive and stereotyped behaviors is only partially understood but the basal gangliafrontal lobe circuitry plays an important role (Langen et al. 2011). Part of this circuitry is regulated by the dopamine system, which may explain the high prevalence of motor rigidity and cognitive rigidity in ASD. The dopamine3-receptor gene (DRD3) has been associated with ASD in a British sample and in a sample from The Netherlands (de Krom et al. 2009). In both samples and in a joint statistical analysis, a significant association between ASD and the SNP rs167771 located in the DRD3 gene was found, which survived statistical correction for multiple testing.

This finding is of particular interest for different reasons. First, the rs167771SNP was recently also found to be related to risperidone-induced extra pyramidal symptoms (EPS) (Gassó et al. 2009), which is important since risperidone together with aripripazole are the only U.S. Food and Drug Administration approved drugs for treatment of stereotyped behavior in ASD. Second, the DRD3 receptor is an important action site for atypical antipsychotic drugs, and a polymorphism of the DRD3 receptor, DRD3 Ser9Gly, is a predictor for clinical improvement with risperidone therapy (Correia et al. 2010). Third, DRD3 is highly expressed in the basal ganglia, most notably the caudate nucleus. Enlargement of the caudate nucleus in ASD is one of the best replicated findings in neuroimaging studies and has been related to behavioral rigidity (Langen et al. 2009).

Following up on our previous study, we now sought to determine whether polymorphisms of rs167771 are associated with stereotyped and repetitive behavior in ASD. 


\section{Methods}

Sample

In a previous study 144 ASD patients were recruited and genotyped as described in the original paper (De Krom et al. 2009). The multidisciplinary evaluation included the Autism Diagnostic Interview-Revised (ADI-R, Rutter et al. 2003) and the Autism Diagnostic Observation Schedule (ADOS, Lord et al. 1999). All individuals met criteria for autism or ASD on these measures or fell at most one point short on one of the domains of the ADI-R. IQ was measured using the Wechsler scales (Wechsler 2005a, b; Kort et al. 2005), Raven's Coloured Progressive Matrices (Raven 1995) or the Peabody Picture Vocabulary Test-III-NL (Dunn et al. 2005). Participants were excluded if chromosomal abnormalities (on karyotype), fragile-X, specific single gene disorders or severe medical illness other than epilepsy were present. Written informed consent was obtained from the participants or their parents and the study was approved by the Ethics committee of the UMC Utrecht. ADI-R items scores were available for 91 patients (74 males, 81\%; 17 females, 19\%). Diagnosis included autism, Asperger syndrome or PDDNOS according to DSM-IV(TR).

\section{Statistical Analyses}

ADI-R data were used to test possible association between repetitive behavior and the rs167771 SNP. The ADI-R consists of three domains of behavior: social interaction, communication and repetitive or stereotyped behavior. Two factors underlie the third domain: Repetitive Sensory and Motor Behaviors (RSMB), and Insistence on Sameness (IS) e.g. resistance to change in routine or environment, rituals or compulsion (Cuccaro et al. 2003). We used these factors in the present study. Behavior in this domain is coded as "ever" (reflecting behavior that occurred at any time during lifespan) and as "current" (reflecting behavior that occurred at the time of measurement). To diminish the number of tests in a small sample and prevent type-I errors, we only compared "current" behavior between groups. The reliability of the current codings is assumed to be better than the ever codings, because the latter refer to a large period of time and are based on parents' recollection of behavior in the past.

The frequency distribution of the rs167771 SNP was as follows: homozygote AA 66\%, heterozygote AG 29\%, homozygote GG 5\%. Due to the small number of individuals carrying two copies of the GG allele $(n=5)$, the GG and AG groups were combined into one group of G-allele carriers.

The factors in the repetitive behavior domain (IS-current; RSMB-current) were not normally distributed. Therefore, non-parametric Mann-Whitney $U$-tests were used to test the differences between the homozygote AA group and the G-risk allele carriers on the factors of the repetitive behavior domain of the ADI-R. In order to investigate whether the allele variant is more strongly associated with repetitive behavior than with other ASD characteristics, the two subgroups were also compared on the social domain of the ADI-R. Analyses were performed using SPSS, version 15 . The tests were two-tailed with a threshold for significance of $p<.05$.

\section{Results}

The mean age in years of the participants at the time of ADI-R measurement was $10.6( \pm 5.7)$ ranging from 4 to 32 years. Table 1 shows the sample characteristics.

Table 2 shows the mean ADI-R factor scores of the two subgroups. Individuals carrying the AA variant of the rs167771 SNP scored significantly higher on the IS-factor (resp $U=590, p<.01$ ), indicating more rigid behavior than individuals carrying one or two copies of the minor G-allele. There was no significant difference between the subgroups on the RSMB-factor. Also, there was no association between allele variant and total score on the social domain of the ADI-R (Fig. 1). The association of IS with the AA variant of rs167771 would not be statistically significant if corrected for multiple testing. The effect size of the IS factor was $r=.3$. An effect size of .3-.5 represents a moderate effect size.

\section{Discussion}

In this study the minor $G$ allele of the rs 167771 SNP in DRD3 was associated with the ADI-R factor "insistence on sameness (IS)", a specific type of repetitive behavior in ASD. Interestingly, having this polymorphism decreases the risk for IS, which is in the opposite direction from what was hypothesized. If this finding replicates, further work will be necessary to understand this seeming paradox. These data seem to support the idea of genetically determined subgroups within the ASD spectrum exhibiting discrete behavioral phenotypes. This may eventually also have pharmacological implications especially when it is taken in consideration that rs167771 SNP was recently also associated with EPS (Gassó et al. 2009).

From a neuro-anatomical perspective the association of rs167771 is of interest. Data from neuroimaging studies suggest that frontal striatal circuitry, where $D R D 3$ is highly expressed, is implicated in ASD and rigid behavior (Langen et al. 2009). This circuitry is also implicated in impulsivity, such as in Attention Deficit and Hyperactivity Disorders (ADHD) and several studies suggest an association between DRD3 and ADHD (Durston et al. 2009). 
Table 1 Age, IQ and ADI-R domain algorithm scores

\begin{tabular}{lll}
\hline & Homozygote AA carriers $(\mathrm{n}=60)$ & G-allele carriers $(\mathrm{GG} / \mathrm{GA})(\mathrm{n}=31)$ \\
\hline Mean $(\mathrm{SD})$ range & $77 / 23$ \\
\% epilepsy & $83 / 17$ & 13 \\
$\%$ caucasian & 8 & 100 \\
$\%$ Autism/Asperger/PDD-NOS & 95 & $67 / 11 / 22$ \\
Age & $65 / 3 / 32$ & $11.1(6.4)$ \\
& $10.4(5.4)$ & $4-31$ \\
Verbal IQ* & $4-32$ & $82.3(29.8)$ \\
& $87.2(27.0)$ & $22-129$ \\
Non-verbal IQ* & $28-143$ & $87.4(29.1)$ \\
Total score social domain ADI-R & $91.8(27.2)$ & $36-155$ \\
& $24-155$ & $21.0(5.2)$ \\
Total score communication domain ADI-R for verbal subjects & $16.2(4.1)$ & $11-27$ \\
& $7-24$ & $16.1(5.3)$ \\
Total score communication domain ADI-R for nonverbal subjects & $11.9(1.6)$ & $7-25$ \\
Total score repetitive and stereotyped domain ADI-R & $10-14$ & $12.3(1.7)$ \\
& $5.3(1.8)$ & $10-14$ \\
\hline
\end{tabular}

* VIQ is missing in 12 subjects, of whom 5 subjects were unable to complete the task. PIQ is missing in 2 subjects

Table 2 Comparison of Insistence on Sameness (IS) and Repetitive Sensory and Motor Behaviors (RSMB) factor scores on the ADI-R according to allelic variants of the rs167771 SNP of the DRS3 gene

\begin{tabular}{lccc}
\hline & Homozygote AA $(\mathrm{n}=60)$ & Risk-allele carriers $(\mathrm{GG}$ and AG) $(\mathrm{n}=31)$ & Mann-Whitney $U p$ \\
\hline IS-current & $2.7(2.0)$ & $1.6(2.0)$ & 0.004 \\
RSMB -current & $2.1(1.7)$ & $2.9(2.7)$ & n.s. \\
Total score on social algorithm domain & $21.4(5.4)$ & $21.0(5.2)$ & n.s. \\
\hline
\end{tabular}

Mean scores and standard deviations between bracelets

Higher scores represent more severe behavioral deficits

Fig. 1 Mean score on Insistence on Sameness (IS current), and Social behavior on the ADI-R according to allelic variants of the rs $167771 \mathrm{SNP}$ of the DRS3 gene. Error bars represent standard error. Higher scores represent more severe behavioral deficits
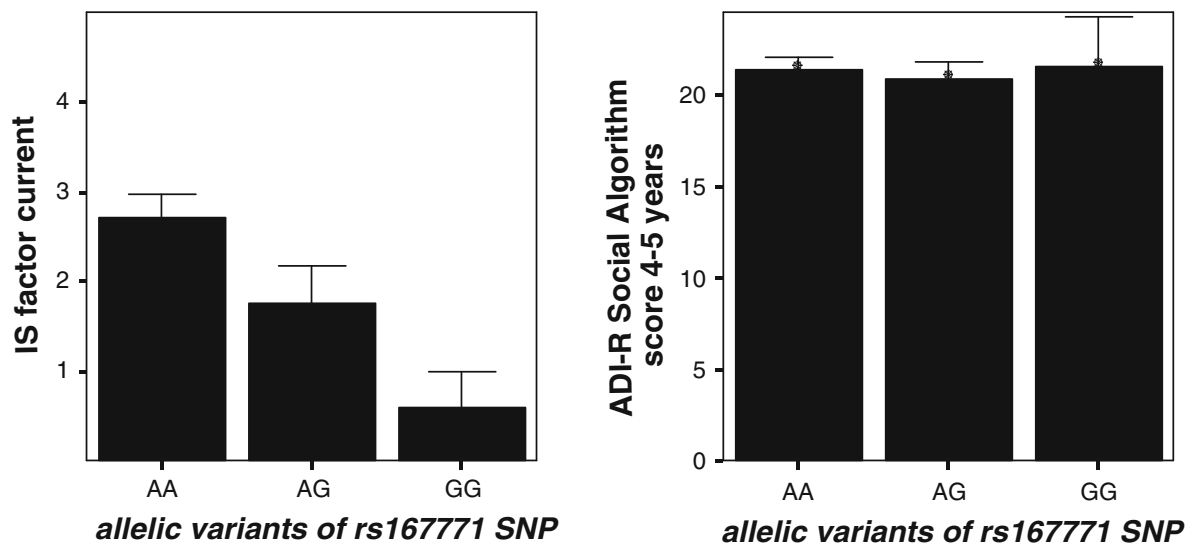

The presented data should be interpreted bearing in mind the relatively small sample size, which is the main limitation of this study. However, the focus on a single SNP has significantly decreased the risk of false positive findings due to multiple testing.
In conclusion, this study suggests that a specific polymorphism of DRD3 is associated with Insistence on Sameness in ASD. This finding obviously needs replication. Nevertheless, together with recent pharmacological findings implicating a role of rs167771 in EPS, these 
findings might contribute to future steps towards personalized pharmacological treatment in ASD.

Acknowledgments The authors thank all subjects for participating in the study and thank Judith Hendriks (Magnus Institute of Neuroscience, Department of Neuroscience and Pharmacology, UMC Utrecht, The Netherlands) for the molecular analysis. Wouter G Staal gratefully acknowledges the Netherlands Brain Foundation for sponsoring his research.

Open Access This article is distributed under the terms of the Creative Commons Attribution Noncommercial License which permits any noncommercial use, distribution, and reproduction in any medium, provided the original author(s) and source are credited.

\section{References}

Correia, C. T., Almeida, J. P., Santos, P. E., Sequeira, A. F., Marques, C. E., Miguel, T. S., et al. (2010). Pharmacogenetics of risperidone therapy in autism: Association analysis of eight candidate genes with drug efficacy and adverse drug reactions. Pharmacogenomics Journal, 10(5), 418-430.

Cuccaro, M. L., Shao, Y., Grubber, J., Slifer, M., Wolpert, C. M., Donnelly, S. L., et al. (2003). Factor analysis of restricted and repetitive behaviors in autism using the Autism Diagnostic Interview-R. Child Psychiatry and Human Development Fall, 34(1), 3-17.

de Krom, M., Staal, W. G., Ophoff, R. A., Hendriks, J., Buitelaar, J., Franke, B., et al. (2009). A common variant in DRD3 receptor is associated with autism spectrum disorder. Biological Psychiatry, 65(7), 625-630.

Dunn, L. M., Dunn, L. M., \& Schlichting, L. (2005). Peabody picture vocabulary test-III-NL handleiding. Amsterdam: Harcourt Assessment B.V.
Durston, S., de Zeeuw, P., \& Staal, W. G. (2009). Imaging genetics in ADHD: A focus on cognitive control. Neuroscience and Biobehavioral Reviews, 33(5), 674-689 (Review).

Gassó, P., Mas, S., Bernardo, M., Alvarez, S., Parellada, E., \& Lafuente, A. (2009). A common variant in DRD3 gene is associated with risperidone-induced extrapyramidal symptoms. Pharmacogenomics Journal, 9(6), 404-410.

Kort, W., Schittekatte, M., Bosmans, M., Compaan, E. L., Dekker, P. H., Vermeir, G., et al. (2005). Wechsler intelligence scale for children-III, Dutch version. Amsterdam: Pearson.

Langen, M., Durston, S., Kas, M. J., van Engeland, H., \& Staal, W. G. (2011). The neurobiology of repetitive behavior: ....and men. Neuroscience and Biobehavioral Reviews, 35(3), 356-365.

Langen, M., Schnack, H. G., Nederveen, H., Bos, D., Lahuis, B. E., de Jonge, M. V., et al. (2009). Changes in the developmental trajectories of striatum in autism. Biological Psychiatry, 66(4), 327-333.

Lord, C., Rutter, M., DiLavore, P. C., \& Risi, S. (1999). ADOS. Autism diagnostic observation schedule. Manual. Los Angeles: WPS.

Parikh, M. S., Kolevzon, A., \& Hollander, E. (2008). Psychopharmacology of aggression in children and adolescents with autism: A critical review of efficacy and tolerability. Journal of Child and Adolescent Psychopharmacology, 18(2), 157-178. (Review).

Raven, J. C. (1995). Colored progressive matrices sets I and II., 1996 edn. Oxford: Oxford Psychologists Press Ltd.

Rutter, M., Le Couteur, A., \& Lord, C. (2003). ADI-R: Autism diagnostic interview-revised manual. Los Angeles, CA: WPS.

Wechsler, D. (2005a). Wechsler adult intelligence scale-III: Dutch version. Amsterdam: Pearson.

Wechsler, D. (2005b). Wechsler Intelligence Scale for Children-III, Dutch Version. Amsterdam: Pearson. 